\title{
On the Efficacy of Static Prices for Revenue Management in the Face of Strategic Customers
}

YIWEI CHEN, Singapore University of Technology and Design

VIVEK F. FARIAS, Massachusetts Institute of Technology

The present paper considers a canonical revenue management problem wherein a monopolist seller seeks to maximize revenues from selling a fixed inventory of a product to customers who arrive over time. We assume that customers are forward looking and strategize on the timing of their purchase, an empirically confirmed aspect of modern customer behavior. In the event that customers were myopic, foundational work by Gallego and van Ryzin [1994] established that static prices were asymptotically optimal for this problem. In stark contrast, for the case where customers are forward looking, available results in mechanism design and dynamic pricing offer no such simple solution and are also constrained by restrictive assumptions on customer type.

The present paper studies the revenue management problem while assuming forward looking customers. We demonstrate that for a broad class of customer utility models, static prices surprisingly continue to remain asymptotically optimal in the scaling regime where inventory and demand grow large. We further show that irrespective of regime, an optimally set static price guarantees the seller revenues that are within at least $63.2 \%$ of that under an optimal dynamic mechanism. The class of customer utility models we consider is parsimonious and enjoys empirical support. It also subsumes many of the utility models considered for this problem in existing mechanism design research; we allow for multi-dimensional customer types. We also allow for a customer's disutility from waiting to be positively correlated with his valuation. Our conclusions are thus robust and provide a simple solution to what is considered a challenging problem of dynamic mechanism design.

CCS Concepts: $\bullet$ Mathematics of computing $\rightarrow$ Stochastic control and optimization; $\bullet$ Theory of computation $\rightarrow$ Dynamic programming; Algorithmic mechanism design; Computational pricing and auctions;

Additional Key Words and Phrases: Mechanism design; revenue management; dynamic pricing; algorithm; strategic customers

\section{REFERENCES}

Guillermo Gallego and Garrett van Ryzin. 1994. Optimal dynamic pricing of inventories with stochastic demand over finite horizons. Management Science 40, 8 (1994), 999-1020.

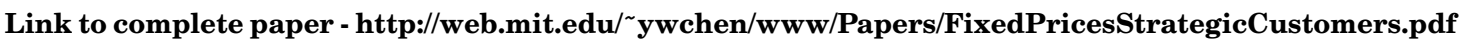

Author's addresses: Yiwei Chen, Engineering Systems and Design, Singapore University of Technology and Design; email: yiwei_chen@sutd.edu.sg; Vivek F. Farias, Sloan School of Management, Massachusetts Institute of Technology; email: vivekf@mit.edu.

Permission to make digital or hard copies of part or all of this work for personal or classroom use is granted without fee provided that copies are not made or distributed for profit or commercial advantage, and that copies bear this notice and the full citation on the first page. Copyrights for third-party components of this work must be honored. For all other uses, contact the owner/author(s). Copyright is held by the author/owner(s).

EC'16, July 24-28, 2016, Maastricht, The Netherlands.

ACM 978-1-4503-3936-0/16/07.

http://dx.doi.org/10.1145/2940716.2940737 